De Tampere à Séville : bilan de la sécurité européenne $(2 / 2)$

\title{
Proposition de décision-cadre du Conseil relative au mandat d'arrêt européen et aux procédures de remise entre Etats membres
}

\section{Commission des communautés européennes}

\section{(2) OpenEdition \\ Journals}

Édition électronique

URL : http://journals.openedition.org/conflits/803

DOI : $10.4000 /$ conflits. 803

ISSN : $1777-5345$

Éditeur :

CCLS - Centre d'études sur les conflits lilberté et sécurité, L'Harmattan

Édition imprimée

Date de publication : 1 juin 2002

ISBN : 2-7475-3030-2

ISSN : 1157-996X

Référence électronique

Commission des communautés européennes, «Proposition de décision-cadre du Conseil relative au mandat d'arrêt européen et aux procédures de remise entre Etats membres », Cultures \& Conflits [En ligne], 46 | été 2002, mis en ligne le , consulté le 30 mars 2021. URL : http://journals.openedition.org/ conflits/803; DOl : https://doi.org/10.4000/conflits.803

Ce document a été généré automatiquement le 30 mars 2021.

Creative Commons License 


\title{
Proposition de décision-cadre du Conseil relative au mandat d'arrêt européen et aux procédures de remise entre Etats membres
}

\author{
Commission des communautés européennes
}

Bruxelles, le 19.9.2001 $\operatorname{COM}(2001) 522$ final

2 2001/0215 (CNS)

\section{EXPOSE DES MOTIFS}

3 1. HISTORIQUE DE L'EXTRADITION

4 L'extradition est née afin de permettre dans un contexte souvent complexe de relations politiques et diplomatiques d'Etat à Etat, la remise aux autorités étrangères, en vue de sa poursuite ou de l'exécution d'une peine, d'un étranger trouvé sur le territoire. Souvent lente et complexe, elle n'est plus adaptée à un espace sans frontière tel que l'espace européen, marqué par un niveau élevé de confiance et de coopération entre Etats qui partagent une conception exigeante de l'Etat de droit.

5 Elle est régie à ce jour par les dispositions de la Convention européenne d'extradition du 13 décembre 1957 (ci-après dénommée Convention de 1957), du protocole additionnel à cette Convention du 15 octobre 1975 (ci-après dénommé protocole de 1975) du second protocole additionnel du 17 mars 1978 (ci-après dénommé protocole de 1978) et de la Convention européenne du 27 janvier 1977 pour la répression du terrorisme (ci-après dénommée Convention terrorisme). Ces textes constituaient certes à l'époque de leur signature un réel progrès, mais représentent aujourd'hui un mécanisme lourd et obsolète au regard de ce que sont devenues les relations entre les Etats membres de l'Union Européenne.

6 La Convention d'application de l'accord Schengen à travers la mise en place du SIS, a créé un dispositif permettant, dans la pratique, d'améliorer considérablement les mécanismes d'information entre les Etats membres relativement aux personnes 
recherchées, et de faciliter les contacts entre autorités nationales lors de l'arrestation d'une personne. Juridiquement toutefois, la Convention Schengen n'apporte rien au regard des mécanismes classiques de l'extradition prévus par la Convention de 1957.

La Convention relative à la procédure simplifiée d'extradition entre les Etats membres de l'Union Européenne du 10 mars 1995 (ci-après dénommée Convention de 1995) ou la Convention relative à l'extradition entre les Etats membres de l'Union Européenne du 27 septembre 1996 (ci-après dénommée Convention de 1996), ont eu pour but d'accélérer et de simplifier les mécanismes de la Convention de 1957, et d'éliminer la plupart des réserves possibles à celle-ci. Elles ne rompent pas toutefois avec le mécanisme, par définition politique et intergouvernemental de l'extradition. En outre, elles n'ont été ratifiées respectivement que par neuf et huit Etats membres.

\section{LES IMPLICATIONS DE LA RECONNAISSANCE MUTUELLE}

En inscrivant la création d'un espace de liberté, de sécurité et de justice au nombre des objectifs de l'Union, le traité d'Amsterdam ouvre la porte à un changement radical de perspective. Lors du Sommet européen de Tampere, les chefs d'Etat et de gouvernement ont indiqué que la reconnaissance mutuelle des décisions de justice devait devenir la pierre angulaire de la coopération judiciaire tant civile que pénale. En matière d'extradition, l'application du principe de reconnaissance mutuelle conduit à ce que chaque autorité judiciaire nationale reconnaisse ipso facto, et moyennant des contrôles minimaux, la demande de remise d'une personne formulée par l'autorité judiciaire d'un autre Etat membre.

\section{LES NOUVEAUX TRAITES BILATERAUX}

11 Parallèlement aux travaux qui se déroulent dans le cadre de l'Union, et eu égard à l'urgence de trouver des réponses efficaces pour lutter contre l'internationalisation croissante de la criminalité, plusieurs Etats membres ont entamé des discussions bilatérales afin de remplacer le mécanisme obsolète de l'extradition par des mécanismes de simple remise aux autorités judiciaires. Ainsi, l'Italie et l'Espagne ont signé entre elles un traité au mois de décembre dernier. Un traité analogue est en cours de préparation entre l'Espagne et le Royaume-Uni.

12 On doit se féliciter de telles initiatives qui soulignent la confiance que s'accordent mutuellement les systèmes judiciaires les Etats membres. Elles prouvent toutefois l'urgence de réformer les mécanismes multilatéraux existants afin d'éviter de complexifier encore, par la mise en place d'accords bilatéraux entre Etats membres, le paysage juridique déjà foisonnant en matière d'extradition.

\section{LE MANDAT D'ARRET EUROPEEN}

\section{4.1. CONTEXTE}

15 Le plan d'action de Vienne invitait les Etats membres à une prompte ratification et mise en œuvre des instruments existant en matière de d'extradition (point $45 \mathrm{c}$ ). Aux termes des conclusions du Conseil Européen de Tampere, « la procédure formelle d'extradition devrait être supprimée entre Etats membres pour les personnes qui tentent d'échapper à la justice après avoir fait l'objet d'une condamnation définitive » et remplacée "par un simple transfèrement des personnes, conformément à l'article 6 du TUE. Il convient également d'envisager des procédures accélérées d'extradition sans préjudice du droit à un procès équitable » (point 35). La Commission est invitée «à faire des propositions à ce sujet, à la lumière de la Convention d'application de Schengen ». 

millénaire en matière de prévention et de contrôle de la criminalité organisée (recommandation 28) qui prévoit que la Commission doit formuler des propositions «prévoyant une extradition accélérée des personnes reconnues coupables qui tentent d'échapper à la justice ». Elle préconise " la possibilité de créer à long terme, un espace juridique européen en matière d'extradition", et d'examiner dans ce contexte "la question de l'extradition dans les procédures par contumace, dans le plein respect des droits fondamentaux garantis par la Convention Européenne des droits de l'homme ». tragiques et meurtrières perpétrées contre le peuple des Etats Unis d'Amérique le 11 septembre 2001, les chefs d'Etat et de gouvernement de l'Union Européenne, la présidente du Parlement Européen, le président de la Commission européenne, et le Haut représentant pour la politique étrangère et de sécurité commune ont appelé « à la création d'un mandat européen d'arrestation et d'extradition conformément aux conclusions de Tampere, et à la reconnaissance mutuelle des décisions de justice ».

Pour l'élaboration de cette proposition, les services de la Commission ont mené une série d'interviews dans les Etats membres avec des praticiens, magistrats, avocats, universitaires, et responsables ministériels spécialistes de l'extradition dans presque tous les Etats membres. Il est apparu au terme de ces échanges qu'il n'y avait aucune raison de distinguer les situations dans lesquelles l'extradition est demandée dans la phase préalable au procès pénal et celles dans lesquelles elle est demandée pour l'exécution de jugements exécutoires. Aucun texte bilatéral ni multilatéral n'introduit cette distinction qui, par ailleurs, ne se justifie pas en pratique. C'est pourquoi, dans un souci de simplification de l'ordre juridique existant, le mandat d'arrêt européen doit couvrir un champ d'application identique à celui de l'extradition auquel il se substitue, et concerner aussi bien la phase pré-sentencielle du procès pénal que la phase postsententielle.

\subsection{CHOIX DE L'INSTRUMENT}

C'est dans un même souci d'efficacité qu'il a été décidé de recourir à une décision-cadre pour la création du mandat d'arrêt européen. Les nombreuses Conventions élaborées dans le cadre du Conseil de l'Europe, de la coopération politique européenne, ou de l'Union européenne ont eu un succès limité comme l'atteste l'état des ratifications. Tant l'ordre juridique résultant du Traité d'Amsterdam que l'état avancé de la coopération judiciaire entre les Etats membres justifie la création du mandat d'arrêt européen par une décision-cadre qui, aux termes de l'article 34 du TUE « lie les Etats membres quand aux résultat à atteindre, tout en laissant aux instances nationales la compétence quant à la forme et aux moyens ».

\subsection{UN INSTRUMENT SOUCIEUX DE LA PROTECTION DES DROITS FONDAMENTAUX}

21 Le système proposé a un double objectif. D'une part, du point de vue de l'efficacité de la répression des activités criminelles, il tire les conséquences de l'ouverture des frontières à l'intérieur de l'espace judiciaire européen en facilitant l'action de la justice de chacun des Etats membres de part et d'autre des frontières. En cela, ce mécanisme est une contribution importante à la lutte contre la criminalité organisée transnationale. D'autre part, ce système répond aux préoccupations des citoyens européens en matière de garantie des droits individuels. 

lautorité judiciaire de l'état d'arrestation devra obligatoirement se prononcer sur le maintien en détention de cette personne en fonction des garanties de représentation qu'elle présente. Si celles-ci sont satisfaisantes, la personne arrêtée pour l'exécution d'un mandat d'arrêt européen pourra être laissée en liberté, le cas échéant sous condition, dans l'Etat d'exécution, jusqu'à être effectivement présentée devant l'autorité judiciaire émettrice à une date utile pour la procédure en cours (article 14). Ce mécanisme devrait éviter des détentions provisoires prolongées, et parfois uniquement dues à l'éloignement géographique de la personne. De même, l'autorité judiciaire émettrice pourra décider de suspendre l'exécution du mandat d'arrêt européen lorsque la personne arrêtée s'engage à se représenter volontairement (article $13(3))$;

3) les personnes ayant fait l'objet d'une décision in absentia devront être jugées de nouveau, après avoir fait opposition devant l'autorité judiciaire d'exécution (article 35) ;

26 4) les cas de détention provisoire prononcés principalement pour s'assurer du maintien à la disposition de la justice de personnes qui résident dans d'autres Etats membres devraient être limités car l'efficacité du mandat d'arrêt européen améliore les garanties de remises et de représentation à l'autorité judiciaire d'émission (article 17) ;

5) des transferts inutiles ou non souhaitables seront évités par le recours à la vidéoconférence (article 34). De même la mise à exécution de la peine dans le lieu où la réinsertion de la personne condamnée se fera dans les meilleurs conditions, devrait être favorisée (articles 33 et 36);

28 6) le cours des procédures pénales sera accéléré, notamment du fait du recours accru au transfèrement temporaire d'un Etat à l'autre (articles 39 et 40), ce qui contribuera à faire respecter le droit des justiciables à bénéficier d'une décision de justice dans un délai raisonnable. Encadrée dans un strict délai de quatre vingt dix jours (article 20), la procédure du mandat d'arrêt européen devrait être une contribution importante au respect du délai raisonnable;

29 7) la suppression du principe de double incrimination ne se fait pas aux dépens des Etats dont la législation est la moins répressive. En effet, le mécanisme de la liste négative permet aux Etats membres qui ont choisi de dépénaliser certains comportements d'exclure ceux-ci du champ d'application du mandat d'arrêt européen (article 27) ;

30 8) la possibilité pour un Etat de soumettre l'exécution d'un mandat d'arrêt européen à la garantie que la peine de détention à perpétuité ne sera pas appliquée est affirmée (article 37).

31 Enfin, et bien sûr, dans l'émission et l'exécution des mandats d'arrêt européens, les juges nationaux restent soumis aux normes générales relatives à la protection des droits fondamentaux, et tout particulièrement à la Convention européenne de sauvegarde des droits de l'homme et des libertés fondamentales de 1950 et à la Charte des droits fondamentaux de l'Union européenne.

\subsection{PRÉSENTATION GENERALE}

Cultures \& Conflits, 46 | été 2002 

conséquence, la phase de recours administratif sur la décision politique, est elle aussi, supprimée. La suppression de ces deux niveaux de procédure devrait considérablement améliorer l'efficacité et la rapidité du dispositif.

40 5) Le mandat d'arrêt européen tiendra compte du principe de la citoyenneté de l'Union. L'exception faite au profit des nationaux ne devrait plus avoir lieu d'être. Le critère le plus pertinent n'est pas la nationalité mais le lieu de résidence principale de la personne, notamment en ce qui concerne l'exécution de la peine. Ainsi, il est envisagé d'une part, de faciliter l'exécution de la peine prononcée dans le pays de l'arrestation lorsque c'est là qu'une bonne réinsertion de la personne est la plus probable, et d'autre part, lorsqu'il sera procédé à l'exécution du mandat d'arrêt européen, de permettre de subordonner celle-ci à la garantie du retour ultérieur de la personne pour l'exécution de la peine prononcée par l'autorité étrangère.

41 6) Les cas de refus d'exécution du mandat d'arrêt sont limités et énumérés afin de simplifier et d'accélérer au maximum la procédure. Le principe de la double incrimination est supprimé de même que le principe de spécialité. En revanche, les Etats membres ont la possibilité, s'ils le souhaitent, d'établir une liste négative d'infractions pour lesquelles ils déclareront refuser l'exécution sur leur territoire de mandats d'arrêt européens. De même, il est possible de rétablir l'exigence de la double 
incrimination pour les cas dans lesquels l'Etat émetteur a exercé une compétence extraterritoriale.

7) Les éléments figurant dans le mandat d'arrêt européen sont uniformisés au niveau de l'Union. Ils devront, sauf cas exceptionnel, permettre à l'autorité du pays d'exécution de procéder à la remise de la personne sans qu'il soit procédé à d'autres contrôles.

8) Le mécanisme du mandat d'arrêt européen a vocation à se substituer, entre Etats membres, à la Convention de 1957, à ses deux protocoles de 1975 et 1978, aux dispositions relatives à l'extradition de la Convention terrorisme, ainsi qu'aux deux Conventions de 1995 et 1996 signées dans le cadre de l'Union. Certaines dispositions de la convention d'application de l'accord Schengen sont également remplacées. (...)

INDEX

Mots-clés : coopération internationale, droit européen, textes et documents officiels 